\title{
Possible role of thymidine phosphorylase in gynecological tumors as an individualized treatment strategy
}

\author{
MASAKO SHIDA $^{1}$, MASANORI YASUDA ${ }^{2}$, MARIKO FUJITA ${ }^{1}$, MASAKI MIYAZAWA ${ }^{1}$, HIROSHI KAJIWARA ${ }^{3}$ \\ TAKESHI HIRASAWA ${ }^{1}$, MASAE IKEDA ${ }^{1}$, NARUAKI MATSUI ${ }^{4}$, TOSHINARI MURAMATSU ${ }^{5}$ and MIKIO MIKAMI ${ }^{1}$ \\ ${ }^{1}$ Department of Obstetrics and Gynecology, Tokai University School of Medicine, Isehara, Kanagawa 259-1193;
${ }^{2}$ Department of Pathology, Saitama Medical University International Medical Center, Hidaka, Saitama 350-1298;
${ }^{3}$ Department of Pathology, Tokai University School of Medicine, Isehara, Kanagawa 259-1193;
${ }^{4}$ Department of Pathology, Tokai University Oiso Hospital, Oiso, Kanagawa 259-0198;
${ }^{5}$ Department of Obstetrics and Gynecology, Tokai University Hachioji Hospital, Hachioji, Tokyo 192-0032, Japan
}

Received March 13, 2015; Accepted April 1, 2016

DOI: $10.3892 / \mathrm{ol} .2016 .5082$

\begin{abstract}
Thymidine phosphorylase (TP) is structurally similar to platelet-derived endothelial cell growth factor, and it activates 5-fluorouracil (5-FU) prodrugs and also promotes angiogenesis. In the present study, the possibility of using TP expression as a biomarker for 5-FU prodrugs, and the significance of TP as an angiogenic factor, were investigated in patients with gynecological tumors. The subjects enrolled in the study were 188 patients with gynecological tumors who provided informed consent and underwent tumor resection at the Department of Obstetrics and Gynecology of Tokai University Hospital between February 2002 and January 2010. Measurement of the enzymatic activity of TP and dihydropyrimidine dehydrogenase (DPD) was performed by enzyme-linked immunosorbent assay. In addition, immunohistochemistry (IHC) analysis of microvessels by monochrome imaging, western blotting and reverse transcription-polymerase chain reaction were performed. The mean TP activity and the TP/DPD ratio were increased in squamous cell carcinoma of the cervix (306.9 and $2.2 \mathrm{U} / \mathrm{mg}$ protein, respectively) and adenosquamous carcinoma (317.6 and $1.4 \mathrm{U} / \mathrm{mg}$ protein, respectively) compared with benign tumors and other malignancies, including endometrial (uterine) carcinoma, ovarian serous adenocarcinoma and ovarian mucinous adenocarcinoma. However, these parameters were also elevated in other histological types of cancer such as clear cell adenocarcinoma of the ovary (115.2 and $2.1 \mathrm{U} / \mathrm{mg}$ protein, respectively), in which the microvessel area was the largest of all the histological types analyzed. Since high TP expression and a high
\end{abstract}

Correspondence to: Professor Masanori Yasuda, Department of Pathology, Saitama Medical University International Medical Center, 1397-1 Yamane, Hidaka, Saitama 350-1298, Japan

E-mail:mmikami0603@gmail.com

Key words: gynecological tumor, thymidine phosphorylase, dihydropyrimidine dehydrogenase, 5-FU prodrug
TP/DPD ratio were identified in other tumors besides cervical cancer, it is possible that patients for whom 5-FU prodrugs are indicated could be selected appropriately if their TP activity is determined and their TP expression is analyzed by IHC prior to initiation of the treatment.

\section{Introduction}

Pyrimidine nucleoside phosphorylase is the collective name for enzymes involved in the metabolism of pyrimidine nucleosides, which convert thymidine to thymine and participate in the biosynthesis of pyrimidine nucleosides, angiogenesis and activation of anti-neoplastic drugs (1). There are two thymidine phosphorylases: Uridine phosphorylase (UP), which belongs to the family of glycosyltransferases, specifically the pentosyltransferases; and thymidine phosphorylase (TP), which belongs to the family of glycosyltransferases, specifically the pentosyltransferases (2). UP has uridine and thymidine as its substrates, and is abundant in mice and rats (2), while TP only has thymidine as its substrate, and is abundant in humans (2). TP is involved in the synthesis of nucleic acids, and its activity is increased in cancer cells due to the additional nucleic acid synthesis required for active cell proliferation (2). TP has a similar structure to that of the angiogenic factor platelet-derived endothelial cell growth factor (PD-ECGF), and promotes vascular endothelial migration (3).

Another tumor-associated action of TP that has attracted attention is the activation of 5-fluorouracil (5-FU) prodrugs (2). 5-FU was first synthesized by Dushinsky et al (4) in 1957, and its efficacy as an anti-cancer agent was subsequently established by the fundamental and clinical studies of Heidelberger et al (5). Since then, its use has been approved for a variety of tumors, including breast and gastrointestinal cancers (6-10). Once incorporated into cells by nucleotide transporters, 5-FU is largely degraded and inactivated by dihydropyrimidine dehydrogenase (DPD), prior to be excreted in the urine as $\alpha$-fluoro- $\beta$-alanine, while unchanged $5-\mathrm{FU}$ is phosphorylated and activated via the same pyrimidine metabolic pathway that processes uracil (11). The anti-neoplastic effect of 5-FU generally depends on the following mechanism: 
When 5-FU is metabolized to fluorodeoxyuridine monophosphate (FdUMP) by TP, it forms a strong ternary complex with thymidylate synthetase and 5,10-methylenetetrahydrofolate (a reduced folic acid coenzyme), thus inhibiting the conversion of dUMP to thymidine 5'-monophosphate and interfering with DNA synthesis (11). 5-FU also causes RNA dysfunction when it is incorporated into intracellular RNA by orotate phosphoribosyltransferase (11). Development of 5-FU prodrugs with various mechanisms of action has enabled the availability of a number of drugs, including doxifluridine, capecitabine, uracil plus tegafur (UFT) and titanium silicate-1 (Fig. 1). These prodrugs are designed to reduce adverse reactions to 5-FU or to exhibit enhanced activity against tumors with elevated TP expression, since these agents display an anti-tumor effect upon being converted to 5-FU by TP in tumor cells (12).

TP is an important enzyme that activates 5-FU. However, there are a limited number of studies on TP expression in gynecological cancer, with the exception of cervical cancer, for which 5-FU prodrugs have already been approved (12), and no studies have been performed to date to compare TP expression among all gynecological tumors. In the present study, the expression of TP was analyzed in various types of gynecological cancer, and the expression of TP in these tumors was compared with the tumor characteristics to explore the possibility of individualized treatment.

\section{Materials and methods}

Patients and tissue specimens. A total of 188 patients who underwent surgery for gynecological tumors at the Department of Obstetrics and Gynecology of Tokai University Hospital (Isehara, Kanagawa, Japan) between February 2002 and January 2010 were enrolled in the present study (Table I). At the time of surgery for the benign tumors, samples that were considered normal tissues, including myometrium, endometrium and ovary in 33 patients, were resected. The samples were confirmed to be free from gynecological neoplasm and pathologically diagnosed as normal tissue. The Institutional Review Board (IRB) for Clinical Research of Tokai University School of Medicine (Isehara, Kanagawa, Japan) approved the present study (IRB no. 09R-082). Written informed consent was obtained from all patients for the use of the resected specimens at the time of enrollment. The present study was performed in accordance with the Declaration of Helsinki. Clinicopathological staging was performed according to the International Federation of Gynecology and Obstetrics classification (13).

Enzyme-linked immunosorbent assay (ELISA) for TP and DPD activity. TP and DPD activities were measured by a sandwich ELISA using a Protein Detector ELISA kit (KPL, Inc., Gaithersburg, MD, USA), according to the manufacturer's protocol. A 96-well plate was incubated for $1 \mathrm{~h}$ at room temperature (RT) with $10 \mu \mathrm{g} / \mathrm{ml}$ monoclonal mouse anti-TP (catalog no., 1C6-203; Roche Diagnostics GmbH, Mannheim, Germany) and $10 \mu \mathrm{g} / \mathrm{ml}$ monoclonal mouse anti-DPD antibodies (Nippon Roche Research Center, Kamakura, Kanagawa, Japan). Then, the plasmatic compartment was added to each well and incubated with the antibodies for $1 \mathrm{~h}$. Upon washing the plate with PBS containing 0.05\% Tween-20 (Wako Pure
Chemical Industries, Ltd., Osaka, Osaka, Japan), incubation was conducted with anti-TP and anti-DPD antibodies overnight at $4^{\circ} \mathrm{C}$. Next, peroxidase-linked species-specific $\mathrm{F}\left(\mathrm{ab}^{\prime}\right)_{2}$ fragments of anti-rabbit immunoglobulin (Ig)G (dilution, 1:10,000; catalog number NA9340; GE Healthcare Life Sciences, Chalfont, UK) were added to each well for $1 \mathrm{~h}$. Subsequently, a reaction was conducted at $\mathrm{RT}$ for 15 min with substrate solution containing 3,3',5,5'-tetramethylbenzidine and $\mathrm{H}_{2} \mathrm{O}_{2}$ (TMB Microwell Peroxidase Substrate system; KPL), and the absorbance at $450 \mathrm{~nm}$ was measured using a microplate reader (3550; Bio-Rad Laboratories, Inc., Hercules, CA, USA).

Immunohistochemistry (IHC). The expression of TP and cluster of differentiation (CD)34 in gynecological tumors was determined by IHC analysis and hematoxylin (Merck, Ltd., Tokyo, Japan) and eosin (Muto Pure Chemicals, Co., Ltd, Tokyo, Japan) staining in all 188 patients. Tumor sections were deparaffinized in xylene ( 3 times for 5 min each) and ethanol (4 times for 5 min each) and heated in $0.01 \mathrm{M}$ citrate buffer (pH 6.0; Dako, Glostrup, Denmark) for $13 \mathrm{~min}$ in a microwave oven at $99^{\circ} \mathrm{C}$. Then, endogenous peroxidase was blocked by incubation in methanol (Wako Pure Chemical Industries, Ltd.) containing $0.3 \% \mathrm{H}_{2} \mathrm{O}_{2}$ (Wako Pure Chemical Industries, Ltd.) for $30 \mathrm{~min}$ at RT. Next, the sections were washed in $0.01 \mathrm{M}$ phosphate-buffered saline (PBS; Wako Pure Chemical Industries, Ltd.) for $10 \mathrm{~min}$, prior to be incubated overnight at $4^{\circ} \mathrm{C}$ with anti-TP (dilution, 1:400) and monoclonal mouse anti-CD34 antibodies (dilution, 1:100; product code, END-L-CE; Novocastra; Leica Microsystems, Ltd., Milton Keynes, UK). Subsequently, the sections were washed in $0.01 \mathrm{M}$ PBS and incubated with goat anti-mouse/rabbit IgG conjugated to a horseradish peroxidase-labeled dextran polymer (EnVision kit; catalog no., K1491; Dako) for $60 \mathrm{~min}$ at RT. Upon being washed 20 times in $0.01 \mathrm{M}$ PBS, the sections were developed in a 3'3-diaminobenzidine solution (Dojindo Laboratories, Kamimashiki, Kumamoto, Japan) containing $0.006 \% \mathrm{H}_{2} \mathrm{O}_{2}$ for 3-5 min at $\mathrm{RT}$, and counterstained with hematoxylin. Protein expression was assessed semiquantitatively as negative ( $0 \%$ positive, 0$)$, weak ( $<10 \%$ positive, $1+)$, intermediate $(10-50 \%$ positive, $2+)$ or strong ( $>50 \%$ positive, $3+$ ).

Analysis of microvessels by monochrome imaging. The distribution of microvessels was analyzed by monochrome imaging of sections immunostained with anti-CD34 antibody and mounted on glass slides (Muto Pure Chemicals, Co., Ltd). Tumor stromal microvessels with a longer and shorter diameter of $\leq 5 \mathrm{~mm}$ were counted in five fields (magnification, x200; Axiophot; Carl Zeiss, Oberkochen, Germany) for each patient, and the area occupied by the vessels was measured. Microvessels were only counted in fields without necrosis that were completely filled by tissue.

Western blotting. To confirm the results of TP expression detected by IHC, western blot analysis was performed in four representative tumors of squamous cell carcinoma (SCC) and clear cell adenocarcinoma (CCA) of ovary (OV). Total protein from whole tissue lysates was separated by electrophoresis on NuPAGE Novex 4-12\% Bis-Tris Protein Gels (1.0 mm; 12-well; Thermo Fisher Scientific, Inc., Waltham, MA, USA) and transferred to Immobilon-P polyvinylidene 


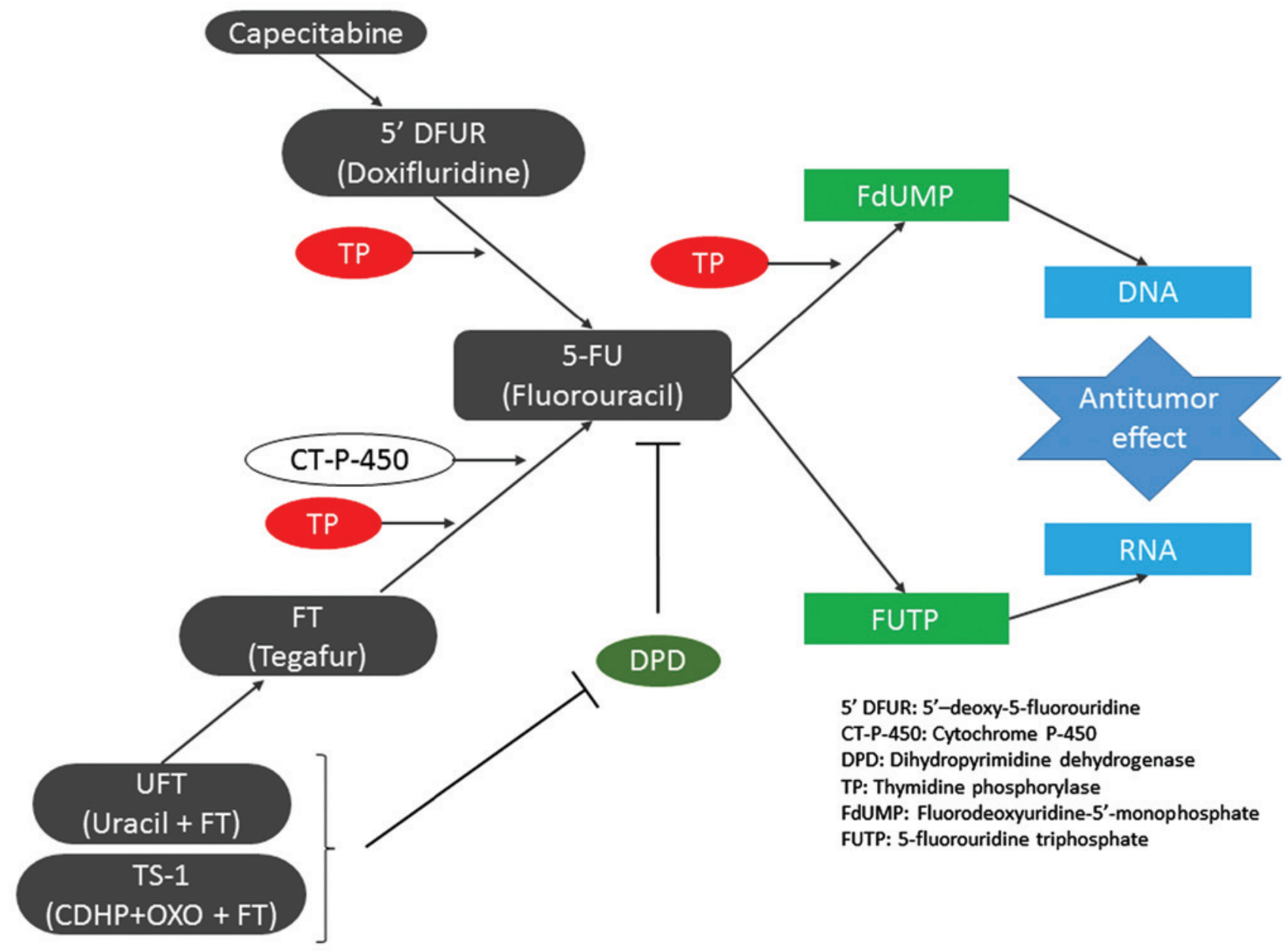

Figure 1. Effects, summary of metabolic pathways and antitumor activity of 5-FU and fluorinated pyrimidines. Capecitabine is absorbed from the gastrointestinal tract, transformed into doxifluridine in the tumor tissue and subsequently transformed into 5-FU by TP to exert an antitumor effect. FT was developed to reduce gastrointestinal toxicity, and is transformed into 5-FU by cytochrome P-450 mainly in the liver and by TP in the tumor tissue, where its concentration remains stable for a long period. Uracil plus FT contains uracil, which has an inhibitory effect on the degradation of 5-FU. TS-1 contains 5-chloro-2,4-dihydroxypyridine, which has a stronger inhibitory effect on dihydropyrimidine dehydrogenase than that of uracil. Oxonate is distributed to the gastrointestinal tract at a high concentration, and reduces gastrointestinal toxicity by inhibiting the phosphorylation of 5-FU. 5-FU is transformed into fluorodeoxyuridine monophosphate in tumor cells, which inactivates TS-1 and inhibits the synthesis of DNA. It also causes dysfunction of RNA through 5-fluorouridine 5'-triphosphate. 5'-DFUR, 5'-deoxy-5-fluorouridine; CT-P-450, cytochrome P-450; DPD, dihydropyrimidine dehydrogenase; TP, thymidine phosphorylase; FdUMP, fluorodeoxyuridine monophosphate; FUTP, 5-fluorouridine 5'-triphosphate; 5-FU, fluorouracil; OXO, oxonate; CDHP, 5-chloro-2,4-dihydroxypyridine; FT, tegafur; UFT, uracil plus tegafur; TS-1, titanium silicate.

difluoride membranes (EMD Millipore, Billerica, MA, USA). The running buffer was 20X NuPAGE MES SDS Running Buffer (Thermo Fisher Scientific, Inc.) and the blotting buffer consisted of Trizma base (Sigma-Aldrich, St. Louis, MO, USA), glycine (Nacalai Tesque, Inc., Kyoto, Kyoto, Japan) and methanol. Precision Plus Protein Standards All Blue (Bio-Rad Laboratories, Inc.) was used as a marker. Membranes were blocked with 5\% skim milk (Wako Pure Chemical Industries, Ltd.) in PBS containing 0.5\% Tween-20 (PBS-T) at RT for $1 \mathrm{~h}$, followed by overnight incubation at $4{ }^{\circ} \mathrm{C}$ with anti-TP antibody diluted 1:500 in PBS-T containing 5\% skim milk. Horseradish peroxidase-conjugated anti-mouse/rabbit complexes were visualized with an ECL Plus kit (GE Healthcare Life Sciences). Cultured HeLa whole-cell lysates served as positive control. The HeLa cells were grown and maintained in $75 \mathrm{~cm}^{2}$ tissue culture flasks in a humidified $5 \% \mathrm{CO}_{2}$ atmosphere at $37^{\circ} \mathrm{C}$. The cells were cultured in Minimum Essential Media (Gibco; Thermo Fisher Scientific, Inc.) supplemented with 10\% heat-incubated fetal bovine serum (Gibco; Thermo Fisher
Scientific, Inc.), $1 \%$ penicillin-streptomycin $(10,000 \mathrm{U} / \mathrm{ml}$ penicillin; 10,000 $\mu \mathrm{g} / \mathrm{ml}$ streptomycin; Gibco; Thermo Fisher Scientific, Inc.).

Reverse transcription-polymerase chain reaction (RT-PCR) analysis. To verify the close association between TP protein and messenger (m)RNA expression in the limited cases of gynecological cancer evaluated in the present study, RT-PCR analysis was performed. TRIzol (500 $\mu \mathrm{l})$ was used to homogenize the samples. Subsequently, the samples were centrifuged at $20,379 \mathrm{x} \mathrm{g}$ and $4^{\circ} \mathrm{C}$ for $15 \mathrm{~min}$, and the aqueous layer was transferred to a different tube. After performing DNase treatment with DNAse I (RQ1 RNase-Free DNase; Promega Corporation, Madison, WI, USA) overnight at $37^{\circ} \mathrm{C}$, the quantity of total RNA dissolved in DEPC-DW was measured using an absorbance measuring instrument, and total RNA was calculated as $5 \mu \mathrm{g}$. Complementary DNA was prepared from four representative tumors using a Ready-To-Go T-primed First-Strand kit (GE Healthcare Life Sciences). The sequences of the TP primers 
Table I. Patient characteristics and TP and DPD activities.

\begin{tabular}{llcccc}
\hline $\begin{array}{l}\text { Classification } \\
\text { (total cases, n=188) }\end{array}$ & \multicolumn{1}{c}{ Histology } & Cases, $\mathrm{n}$ & $\begin{array}{c}\text { TP average, U/mg } \\
\text { protein (SD) }\end{array}$ & $\begin{array}{c}\text { DPD average, U/mg } \\
\text { protein (SD) }\end{array}$ & $\begin{array}{c}\text { TP/DPD } \\
\text { ratio }\end{array}$ \\
\hline Uterine cervical tumors (18) & SCC & 11 & $306.9(106.9)$ & $138.2(66.6)$ & 2.2 \\
& AA & 2 & $317.6(183.7)$ & $222.4(205.3)$ & 1.4 \\
& MUA & 2 & $52.7(1.2)$ & $17.8(15.0)$ & 3.0 \\
Uterine body tumors (53) & SMCC & 3 & $23.1(8.0)$ & $10.4(2.6)$ & 2.2 \\
& EMA-G1 & 22 & $68.6(30.0)$ & $48.7(40.0)$ & 1.4 \\
& EMA-G2 & 5 & $63.3(61.6)$ & $42.1(33.1)$ & 1.5 \\
& EMA-G3 & 6 & $63.0(79.3)$ & $34.6(20.2)$ & 1.8 \\
& SEA & 2 & $52.4(19.2)$ & $45.9(21.9)$ & 1.2 \\
& Adenomyosis & 2 & $26.2(15.6)$ & $33.1(10.6)$ & 0.8 \\
& Leiomyoma & 14 & $12.9(7.7)$ & $48.1(17.5)$ & 0.3 \\
& Leiomyosarcoma & 2 & $41.1(21.4)$ & $54.9(26.9)$ & 0.7 \\
& SEA & 9 & $98.6(65.8)$ & $82.7(70.2)$ & 1.2 \\
& CCA & 16 & $115.2(59.3)$ & $54.2(109.6)$ & 2.1 \\
& MUA & 16 & $54.4(44.4)$ & $63.3(47.1)$ & 0.9 \\
& EMA & 8 & $74.8(47.9)$ & $37.2(12.8)$ & 2.0 \\
& Serous BT & 1 & 42.2 & 59.1 & 0.7 \\
& Mucinous BT & 19 & $17.3(10.4)$ & $52.4(24.2)$ & 0.3 \\
& Mucinous adenoma & 6 & $21.0(16.9)$ & $58.7(19.2)$ & 0.4 \\
& Yolk sac tumor & 4 & $24.4(17.6)$ & $73.2(84.5)$ & 0.3 \\
& Dysgerminoma & 2 & $109.5(41.2)$ & $41.9(1.2)$ & 2.6 \\
& Endometriosis cyst & 1 & 77.5 & 121.8 & 0.6 \\
& Mature teratoma & 1 & 69.5 & 84.7 & 0.8 \\
& Adenofibroma & 1 & 6.4 & 142.9 & 0.0 \\
& Myometrium & 12 & $32.4(38.4)$ & $62.5(19.5)$ & 0.5 \\
& Endometrium & 8 & $27.5(35.2)$ & $40.5(16.3)$ & 0.7 \\
Ovary & 13 & $19.5(14.7)$ & $89.3(55.3)$ & 0.2 \\
\hline
\end{tabular}

SCC, squamous cell carcinoma; AA, adenosquamous carcinoma; MUA, mucinous adenocarcinoma; SMCC, small cell carcinoma; EMA, endometrioid adenocarcinoma; SEA, serous adenocarcinoma; CCA, clear cell adenocarcinoma; BT, borderline tumor; TP, thymidine phosphorylase; DPD, dihydropyrimidine dehydrogenase.

used were as follows: 5'-CTGCTGTATCGTGGGTCAGA-3' and 5'-CAGCGTCTTTGCCAGCTC-3' (Greiner Bio-One International $\mathrm{GmbH}$, Tokyo, Japan). In addition, $\beta$-actin was amplified with another pair of primers (5'-TCATGAAGTGTG ACGTTGACATCCGT-3' and 5'-CCTAGAAGCATTTGCGGT GCACGATG-3'; Promega Corporation), and served as internal standard. PCR was initiated with denaturation at $95^{\circ} \mathrm{C}$ for $10 \mathrm{~min}$, followed by 30 cycles of $95^{\circ} \mathrm{C}$ for $20 \mathrm{sec}, 59^{\circ} \mathrm{C}$ for $30 \mathrm{sec}$ and $72^{\circ} \mathrm{C}$ for $60 \mathrm{sec}$, with a final elongation at $72^{\circ} \mathrm{C}$ for $10 \mathrm{~min}$. PCR products were analyzed by $2 \%$ agarose gel electrophoresis (Wako Pure Chemical Industries, Ltd.), and the gels were stained with ethidium bromide (Invitrogen; Thermo Fisher Scientific, Inc.). Tris-acetate-EDTA (50X; Sigma-Aldrich) was used as the running buffer and $100 \mathrm{bp}$ DNA Ladder (New England Biolabs Japan, Inc., Tokyo, Japan) was used as a marker. Mupid (Advance Co., Ltd., Tokyo, Japan) was used for electrophoresis. Visualization was performed using High Performance UV Transilluminator (UVP, Inc., Upland, CA, USA). Leiomyoma samples were used as negative controls and an RT- control (no reverse transcription) was also performed.
Statistical and prognosis analysis. The overall survival ratios were calculated by the Kaplan-Meier method, and the significance of difference in survival was analyzed by the log-rank test. Quantitative variables are presented as the mean \pm standard deviation. Data were analyzed using SPSS software version 21.0 (IBM SPSS, Armonk, NY, USA). $\mathrm{P}<0.05$ was considered to indicate a statistically significant difference.

\section{Results}

$T P$ and DPD activity in various tumors

Cervical tumors. The mean TP activity was elevated in SCC (306.9 U/mg protein) and adenosquamous carcinoma (AA) (317.6 U/mg protein) of the cervix, although it was not very high in mucinous adenocarcinoma (MUA) $(52.7 \mathrm{U} / \mathrm{mg}$ protein) or small cell carcinoma (SMCC) (23.1 U/mg protein). The mean DPD activity was also elevated in SCC (138.2 $\mathrm{U} / \mathrm{mg}$ protein) and AA (222.4 U/mg protein). However, the TP/DPD ratios of all cervical tumors did not exhibit major differences ( 2.2 for SCC, 1.4 for AA, 3.0 for MUA and 2.2 for SMCC). 

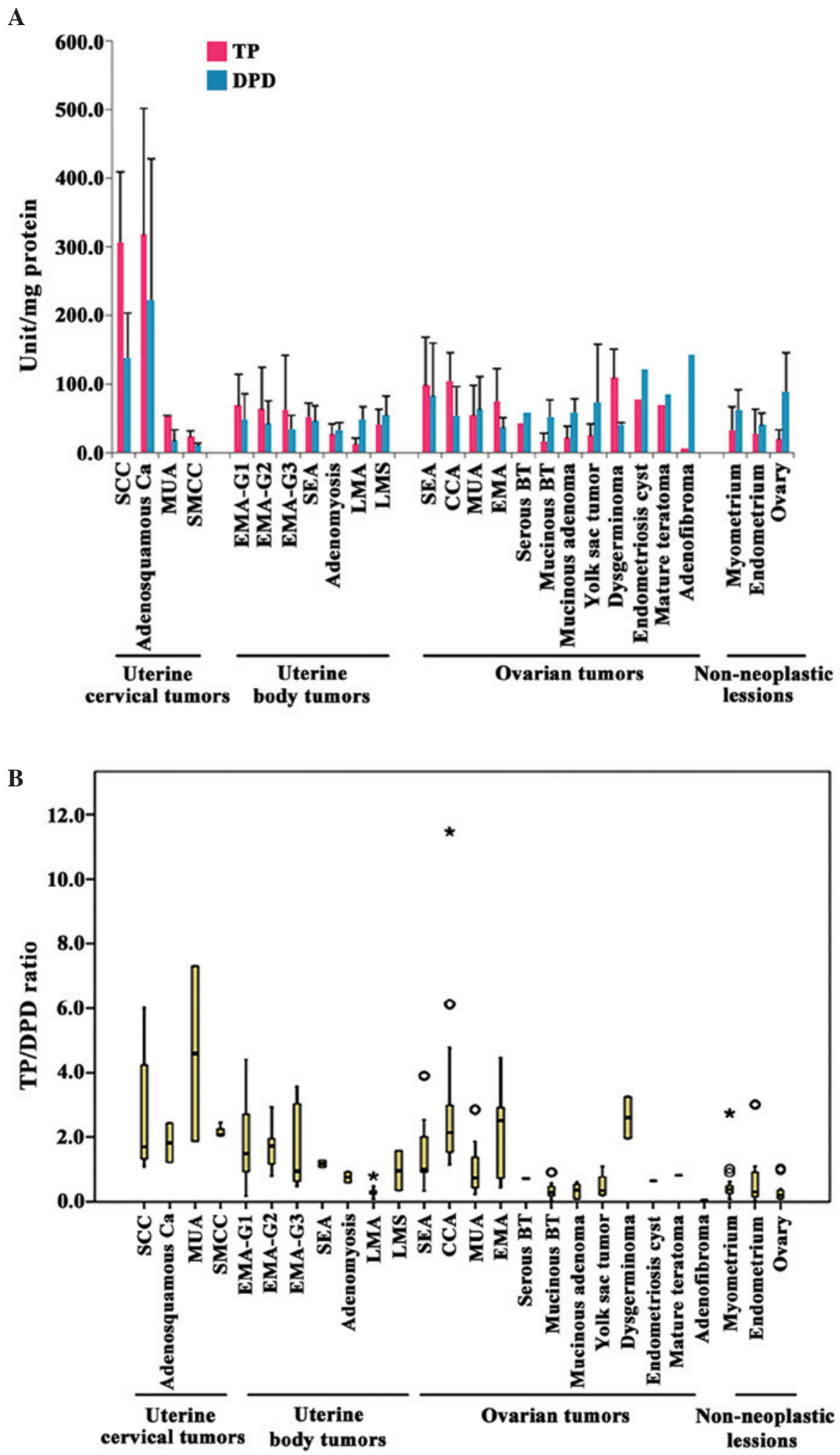

Figure 2. (A) Enzymatic activity of TP and DPD (mean levels stratified by histological type) and (B) TP/DPD ratio in gynecological tumors. The mean enzymatic activity of TP and DPD was higher in SCC of the cervix and adenosquamous carcinoma compared with other tumor types. In patients with tumors of other histological types, the TP levels were often higher than the DPD levels. As observed in SCC of the cervix, the median TP/DPD ratio was also high in other malignant tumors, and a number of patients exhibited a very high TP/DPD ratio. "Outlier point $\geq 3 \times$ IQR; ${ }^{\circ}$ Outlier point $1.5 \times$ IQR $-3 \times$ IQR. SCC, squamous cell carcinoma; AA, adenosquamous carcinoma; MUA, mucinous adenocarcinoma; SMCC, small cell carcinoma; EMA, endometrioid adenocarcinoma; SEA, serous adenocarcinoma; LMA, leiomyoma; LMS, leiomyosarcoma; CCA, clear cell adenocarcinoma; BT, borderline tumor; TP, thymidine phosphorylase; DPD, dihydropyrimidine dehydrogenase; IQR, interquartile range.

Tumors of the uterine corpus. There were no significant differences in TP activity among the different grades of endometrioid adenocarcinoma (EMA), with the mean TP activity being 68.6, 63.3 and $63.0 \mathrm{U} / \mathrm{mg}$ protein in $\mathrm{G} 1, \mathrm{G} 2$ and $\mathrm{G} 3$ tumors, respectively. In contrast, DPD activity decreased as EMA became progressively less differentiated, with the mean 
levels being 48.7, 42.1 and $34.6 \mathrm{U} / \mathrm{mg}$ protein in G1, G2 and G3 tumors, respectively. DPD activity was significantly higher than TP activity in leiomyoma ( $\mathrm{P}=0.048)$, where the mean TP activity was $12.9 \mathrm{U} / \mathrm{mg}$ protein, while the mean DPD activity was $48.1 \mathrm{U} / \mathrm{mg}$ protein, with a TP/DPD ratio of 0.3 . DPD activity was also higher than TP activity in leiomyosarcoma, with a TP/DPD ratio of 0.7 .

Ovarian tumors. Comparison of the TP/DPD ratios among four histological types of epithelial ovarian cancer [serous adenocarcinoma (SEA)-OV, CCA-OV, EMA-OV and MUA-OV] revealed that the TP/DPD ratio was highest for CCA-OV (2.1), followed by EMA-OV (2.0) and SEA-OV (1.2). Mean TP activity was the highest in CCA-OV (115.2 U/mg protein), followed by SEA-OV (98.6 U/mg protein) and EMA-OV (74.8 U/mg protein) and MUA-OV (54.4 U/mg protein). Among non-epithelial ovarian tumors, the mean TP activity was $109.5 \mathrm{U} / \mathrm{mg}$ protein in dysgerminoma, where the TP/DPD ratio was also high (2.6), while the TP/DPD ratios of the other non-epithelial tumors were $\leq 1.0$.

Non-tumor tissues. In normal tissues, including myometrium, endometrium and ovary, the mean DPD activity (64.4 U/mg protein) was higher than the mean TP activity (19.7 U/mg protein), and the TP/DPD ratio was $\leq 0.7$ (Table I and Fig. 2).

Prognosis analysis. The ovarian tumors with a TP/DPD ratio $>1.0$ demonstrated poor prognosis $(\mathrm{P}=0.161$; Fig. 3 ). There were no significant differences among uterine cervical tumors and uterine body tumors (data not shown).

IHC analysis of TP expression and localization. TP expression was observed in a wide range of uterine tumors, but it was generally strongest in MUA, SCC and AA.

In tumors of the uterine corpus, TP expression was strongest in G1 EMA, and TP expression decreased as these tumors became less differentiated. There were significant differences in TP expression among G1, G2 and G3 tumors $(\mathrm{P}<0.01)$. TP expression could not be detected by IHC in $>50 \%$ of leiomyoma cases.

Among ovarian tumors, ELISA also revealed high levels of TP expression in EMA-OV,CCA-OV and SEA-OV. In CCA-OV, TP expression was detected in both the nucleus and cytoplasm of tumor cells, and was stronger than in the other histological types. TP expression was not observed in the normal tissues of the majority of patients. Although TP was predominantly localized to the cytoplasm, it was often detected in the nucleus when the tumor exhibited strong expression (Figs. 4 and 5).

Monochrome imaging of microvessel distribution. When the area occupied by microvessels in a section was measured in each type of ovarian cancer, the part of the vasculature defined as microvessels had an area of $116.6 \mu \mathrm{m}^{2}$ in CCA-OV, $71.8 \mu \mathrm{m}^{2}$ in SEA-OV and $56.8 \mu \mathrm{m}^{2}$ in EMA-OV (Table II and Fig. 6). There were no significant differences among uterine cervical tumors and uterine body tumors (data not shown).

Western blotting analysis. Western blotting revealed dense bands for TP protein expression in patients with SCC and CCA-OV, who exhibited high TP activity and TP positive reaction on immunostaining (Fig. 7).

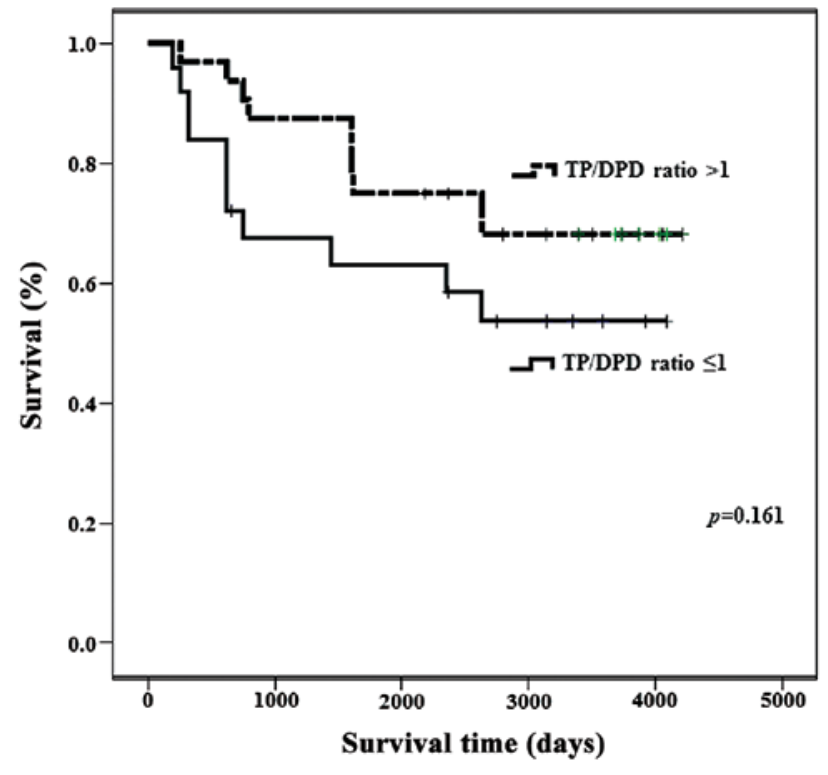

Figure 3. Prognosis analysis. Ovarian tumors with a thymidine phosphorylase/dihydropyrimidine dehydrogenase ratio $>1.0$ exhibited poor prognosis $(\mathrm{P}=0.161)$. TP, thymidine phosphorylase; $\mathrm{DPD}$, dihydropyrimidine dehydrogenase.

RT-PCR analysis. Analysis of TP mRNA expression in patients with prominent TP protein bands in western blot analysis revealed that TP mRNA expression was closely associated with TP protein expression (Fig. 7).

\section{Discussion}

TP activity, which was the focus of the present study, could be measured in fresh tissue specimens and serum, and could also be evaluated by IHC. Therefore, the efficacy of 5-FU prodrugs may be predicted if the activity and expression of TP are investigated prior to initiation of the treatment. While TP activity is known to be increased in cervical cancer (14), the present study demonstrated that TP activity is also high in certain patients with CCA-OV and malignant epithelial ovarian tumors.

DPD acts to degrade TP, and may weaken the therapeutic effect of oral pyrimidine fluoride-based drugs (5). UFT, which contains an oral pyrimidine fluoride-based drug combined with uracil to inhibit DPD, was designed to overcome this problem, even when both TP and DPD activities are high in a tumor $(15,16)$. UFT is a 5-FU prodrug that was developed in Japan as a catabolic enzyme for tegafur and 5-FU (16). Since the response rate to this drug was high $(16 \%)$ in a previous phase II study performed in 25 patients with advanced/recurrent cervical cancer, the use of UFT for the treatment of cervical cancer is already covered by national health insurance in Japan (14). The usefulness of UFT as adjuvant chemotherapy for various other types of cancer has also been established (6-9). These findings suggest that UFT may be useful as adjuvant chemotherapy for gynecological tumors. In a previous phase III study comparing radiotherapy combined with low- or high-dose Z-100 and maintenance therapy, oral pyrimidine fluoride-based drugs such as 5-FU and UFT were used as adjuvant chemotherapy, and comparison was 

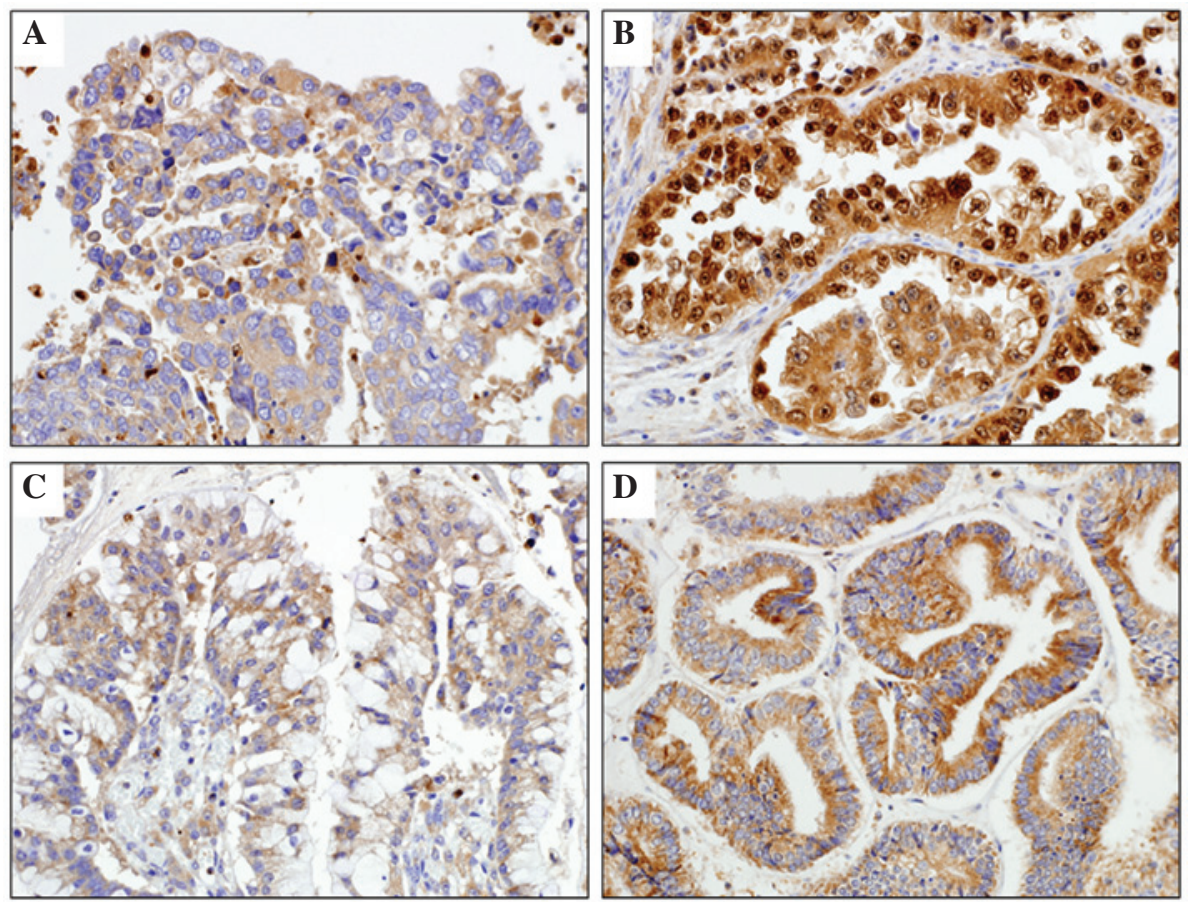

Immunohistochemical expression of TP in ovarian adenocarcinoma (x200)

Figure 4. Immunohistochemical analysis of TP expression in various types of ovarian adenocarcinoma. The expression levels of TP varied among (A) serous adenocarcinoma-OV, (B) mucinous adenocarcinoma-OV, (C) CCA-OV and (D) endometrioid adenocarcinoma-OV. TP was detected both in the nucleus and cytoplasm of tumor cells, particularly in CCA-OV, and stronger staining was observed in CCA-OV than in the other histological types analyzed. Magnification, x200. TP, thymidine phosphorylase; CCA, clear cell adenocarcinoma; OV, of ovary.
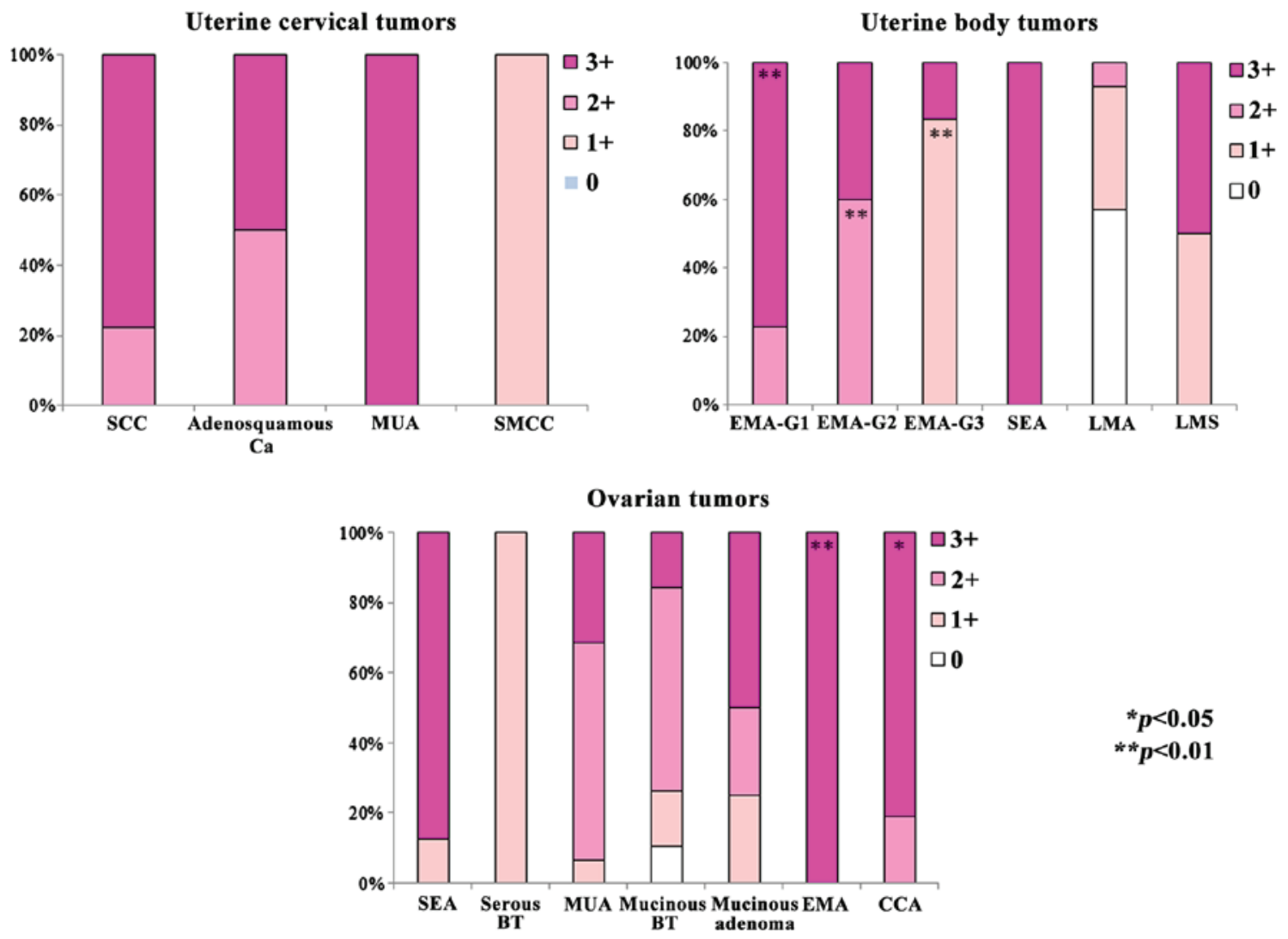

${ }^{*} p<0.05$
${ }^{* *} \boldsymbol{p}<0.01$

Figure 5. Comparison of immunohistochemical findings. All malignant tumors exhibited expression of TP at different levels, whereas benign tumors were often negative for TP expression. Significant differences in positive expression rate were observed among G1, G2 and G3 EMA tumors of the uterus ( $\left.{ }^{* *} \mathrm{P}<0.01\right)$, Strong TP expression (3+) was detected in numerous patients with ovarian tumors such as clear cell adenocarcinoma-OV and EMA-OV ("P $\left.<0.05,{ }^{* *} \mathrm{P}<0.01\right)$. SCC, squamous cell carcinoma; AA, adenosquamous carcinoma; MUA, mucinous adenocarcinoma; SMCC, small cell carcinoma; EMA, endometrioid adenocarcinoma; SEA, serous adenocarcinoma; LMA, leiomyoma; LMS, leiomyosarcoma; BT, borderline tumor; CCA, clear cell adenocarcinoma; TP, thymidine phosphorylase; OV, of ovary. 
Table II. Analysis of vascularity on monochrome images of ovarian carcinoma.

\begin{tabular}{lc}
\hline Histology & Microvessel area, $\mu \mathrm{m}^{2}$ \\
\hline CCA-OV & 116.6 \\
SEA-OV & 71.8 \\
EMA-OV & 56.8 \\
MUA-OV & 43.6 \\
\hline
\end{tabular}

CCA, clear cell adenocarcinoma; SEA, serous adenocarcinoma; EMA, endometrioid adenocarcinoma; MUA, mucinous adenocarcinoma; OV, of ovary.

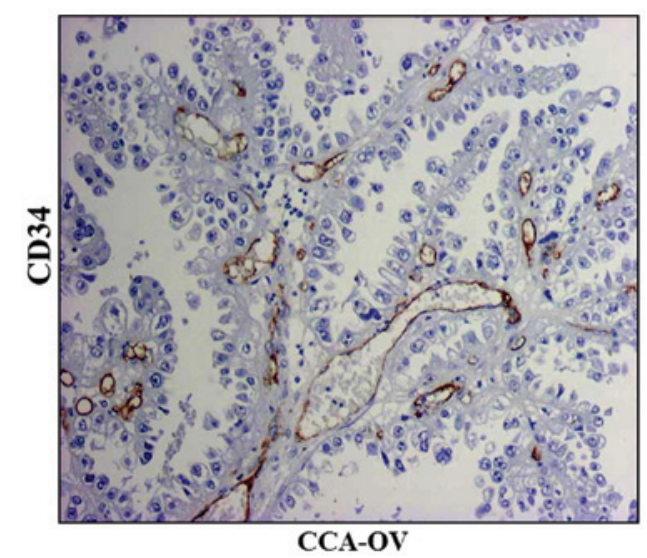

Figure 6. Immunohistochemical expression of CD34 in CCA and analysis of vascularity on monochrome images of ovarian carcinoma. Evaluation of the total microvascular area within tumors revealed that it was largest in clear cell adenocarcinoma-OV, followed by serous adenocarcinoma-OV, endometrioid adenocarcinoma-OV and mucinous adenocarcinoma-OV. Magnification, x200. OV, of ovary; CD, cluster of differentiation; CCA, clear cell adenocarcinoma.

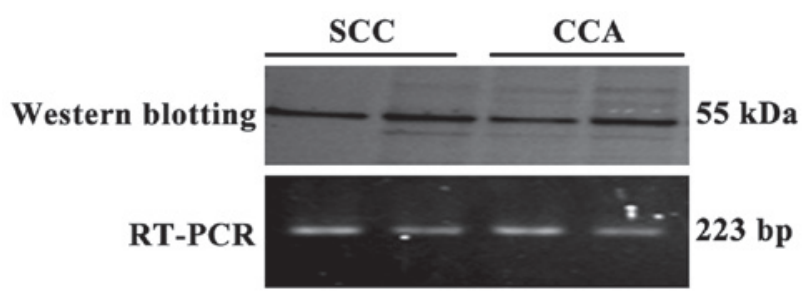

Figure 7. Western blot and reverse transcription-polymerase chain reaction analyses. Western blotting revealed a strong band corresponding to TP protein expression in two patients with squamous cell carcinoma and two patients with clear cell adenocarcinoma of ovary, in whom immunohistochemical expression of TP was also high. Expression of TP messenger RNA was also detected in these patients. SCC, squamous cell carcinoma; CCA, clear cell adenocarcinoma; RT-PCR, reverse transcription-polymerase chain reaction; TP, thymidine phosphorylase.

performed with patients not receiving adjuvant chemotherapy. In that study, the 3- and 5-year survival rates were respectively 22.5 and $15.8 \%$ higher in the adjuvant chemotherapy group than in the group without adjuvant chemotherapy (17). Based on those results, a phase III, randomized, comparative study of UFT in patients with locally advanced cervical cancer receiving radical radiotherapy (termed LUFT trial) is currently ongoing in Japan, whereby radiotherapy and chemotherapy are administered simultaneously to patients with stage IB2-IVA cervical cancer. By July 2014, 180 subjects were enrolled in that study, whose results have not been published thus far. However, exploration of biomarkers was not performed during the LUFT trial (18).

It is known that TP activates 5-FU prodrugs and is also structurally similar to PD-ECGF, an angiogenic factor that enhances the migration of vascular endothelial cells (19). In the present study, IHC demonstrated that TP was strongly positive in three types of epithelial ovarian cancer, including CCA-OV, SEA-OV and EMA-OV (Figs. 4 and 5). Suzuki et al (20) reported that TP was probably closely associated with the mechanism of angiogenesis in CCA-OV, unlike MUA-OV, since TP expression was higher in this type of adenocarcinoma than in other histological types, and was significantly lower in MUA-OV than in other histological types. However, TP expression in different types of cancer remains a matter of controversy. For example, TP expression was reported to be low in CCA-OV, but high in MUA-OV (19), while another study demonstrated high TP expression in SEA-OV and EMA-OV, but not in CCA-OV (21). It was observed in the present study that TP activity could vary considerably within each tumor, and TP expression may depend on the local growth pattern characterized by the histological structure, including papillary, tubular and solid nests. At present, there is little information concerning the association between TP expression and prognosis, due to the insufficient number of patients and duration of observations. However, Fujimoto et al (21) reported that the treatment outcome was significantly worse for ovarian cancer patients with high TP expression than for those with low TP expression. In the present study, the ovarian tumors with a TP/DPD ratio $>1.0$ presented poor prognosis (Fig. 3).

The present study also revealed that TP expression was increased compared with other histological types, and the number of microvessels was larger than other histological types, in CCA-OV and SEA-OV patients, presumably because papillary proliferation is characteristic of these two tumors (22). Ogawa et al (23) also reported that the prognosis was better for CCA-OV patients with a high microvessel density than those with a low microvessel density. Since the present study also demonstrated that CCA-OV tumors exhibited the largest vascular area (Fig. 6) of all the gynecological tumors analyzed, the association between TP expression and angiogenesis or long-term prognosis will be further analyzed in future studies.

The localization of TP was investigated by IHC in the present study, and TP expression was detected in both tumor cells and stromal cells. It has been previously reported that the production of TP by colorectal cancer cells is limited, with the majority of TP being produced by stromal cells around the tumor, particularly activated macrophages (24). It has also been reported that the number of $\mathrm{TP}^{+}$activated macrophages is positively correlated with the number of stromal microvessels, and that activated macrophages producing TP are involved in angiogenesis in colorectal cancer (24). In addition, Konishi (25) reported that TP expression was more common in the stroma of ovarian cancer than in tumor cells, and was closely correlated with the presence of $\mathrm{CD}^{+} 8^{+}$cells and microvessels, particularly at sites of cancer cell infiltration or metastasis associated with a stromal reaction and angiogenesis (25). These findings 
suggest that macrophages may be activated by the tumor cells via certain mechanism to increase $\mathrm{TP}$ expression and promote angiogenesis. Thus, the significance of TP expression may depend on its location (in the tumor cells or in the stroma, particularly at sites of infiltration). Accordingly, the significance of TP localization in patients with gynecological tumors requires further investigation.

In recent years, attempts have been made to transiently increase TP activity by administration of cytokines or other anti-neoplastic drugs, so that pyrimidine fluoride-based drugs could also be used in patients with low TP activity (26). As a result, drugs that inhibit angiogenesis by directly blocking TP activity have been developed $(27,28)$. Phase I clinical studies of these drugs in patients with colorectal and breast cancer have already commenced in the USA (29). While awaiting the results of these studies, research on the development of novel treatments must continue, focusing on TP activity, the significance of TP as a biomarker and the utility of UFT maintenance therapy for gynecological cancer.

\section{References}

1. Haraguchi M, Miyadera K, Uemura K, Sumizawa T, Furukawa T, Yamada K, Akiyama S and Yamada Y: Angiogenic activity of enzymes. Nature 368: 198, 1994.

2. Miwa M, Ura M, Nishida M, Sawada N, Ishikawa T, Mori K, Shimma N, Umeda I and Ishitsuka H: Design of a novel oral fluoropyrimidine carbamate, capecitabine, which generates 5 -fluorouracil selectively in tumours by enzymes concentrated in human liver and cancer tissue. Eur J Cancer 34: 1274-1281, 1998.

3. Furukawa T, Yoshimura A, Sumizawa T, Haraguchi M, Akiyama S, Fukui K, Ishizawa M and Yamada Y: Angiogenic factor. Nature 356: 668, 1992.

4. Dushinsky R, Pleven E and Heidelberger C: The synthesis of 5-fluoropyrimidines. J Am Chem Soc 79: 4559-4560, 1957.

5. Heidelberger C, Chaudhuri NK, Danneberg P, Mooren D, Griesbach L, Duschinsky R, Schnitzer RJ, Pleven E and Scheiner J: Fluorinated pyrimidines, a new class of tumour-inhibitory compounds. Nature 179: 663-666, 1957.

6. Nakajima T, Kinoshita T, Nashimoto A, Sairenji M, Yamaguchi T, Sakamoto J, Fujiya T, Inada T, Sasako M and Ohashi Y; National Surgical Adjuvant Study of Gastric Cancer Group: Randomized controlled trial of adjuvant uracil-tegafur versus surgery alone for serosa-negative, locally advancer gastric cancer. Br J Surg 94: $1468-1476,2007$.

7. Kato H, Ichinose $\mathrm{Y}$, Ohta M, Hata E, Tsubota N, Tada H, Watanabe Y, Wada H, Tsuboi M, Hamajima N and Ohta M; Japan Lung Cancer Research Group on Postsurgical Adjuvant Chemotherapy: A randomized trial of adjuvant chemotherapy with uracil-tegafur for adenocarcinoma of the lung. N Engl J Med 350: 1713-1721, 2004.

8. Akasu T, Moriya Y, Ohashi Y, Yoshida S, Shirao K and Kodaira S; National Surgical Adjuvant Study of Colorectal Cancer: Adjuvant chemotherapy with uracil-tegafur for pathological stage III rectal cancer after mesorectal excision with selective lateral pelvic lymphadenectomy: A multicenter randomized controlled trial. Jpn J Clin Oncol 36: 237-244, 2006.

9. Kato T, Ohashi Y, Nakazato H, Koike A, Saji S, Suzuki H, Takagi H, Nimura Y, Hasumi A, Baba S, et al: Efficacy of ora UFT as adjuvant chemotherapy to curative resection of colorectal cancer: Multicenter prospective randomized trial. Langenbecks Arch Surg 386: 575-581, 2002.

10. Kasumi F, Yoshimoto M, Uchino J, Abe R, Nomura Y, Sugimachi K, Nakazato $\mathrm{H}$ and Abe O: Meta-analysis of five studies on tegafur plus uracil (UFT) as post-operative adjuvant chemotherapy for breast cancer. Oncology 64: $146-153,2003$

11. Hirashima Y and Shirao K: Predicting drug efficacy-fluorinated pyrimidines (fluorouracil, S-1 and capecitabine). Gan To Kagaku Ryoho 39: 1603-1607, 2012 (In Japanese).
12. Yamamoto K, Noda K, Hatae M, Kudo T, Hasegawa K, Nishimura R, Honjo H, Yajima A, Sato S, Mizutani K, et al: Effects of concomitant use of doxifluridine, radiotherapy and immunotherapy in patients with advanced cervical cancer. Oncol Rep 8: 273-277, 2001.

13. Piver MS, Rose PG, Freedman MF: Change in International Federation of Gynecology and Obstetrics staging. Am J Obstet Gynecol. 1988 Mar;158 (3 Pt 1):678-9.

14. Noda K, Teshima K, Ikeda M, Sugawa T, Yamagata S, Sekiba K, Kohno I, Kaneshige E, Sawaragi I, Matsuoka I, et al: Phase II study of UFT in cancer of the uterine cervix. Gan To Kagaku Ryoho 12: 900-906, 1985 (In Japanese).

15. Fujii S, Kitano S, Ikenaka K and Shirasaka T: Effect of coadministration of uracil or cytosine on the anti-tumor activity of clinical doses of 1-(2-tetrahydrofuryl)-5-fluorouracil and level of 5-fluorouracil in rodents. Gan 70: 209-214, 1979.

16. Ikenaka K, Shirasaka T, Kitano S and Fujii S: Effect of uracil on metabolism of 5-fluorouracil in vitro. Gan 70: 353-359, 1979.

17. Noda K, Ohashi Y, Sugimori H, Ozaki M, Niibe H, Ogita S, Kohno I, Hasegawa K, Kikuchi Y, Takegawa Y, et al: Phase III double-blind randomized trial of radiation therapy for stage IIIb cervical cancer in combination with low- or high-dose Z-100: Treatment with immunomodulator, more is not better. Gynecol Oncol 101: 455-463, 2006.

18. Gynecologic Oncology Trial and Investigation Consortium: LUFT Trial: A randomized phase III trial of long UFT administration following curative radiation therapy for locally advanced cervical cancer. Version 1.20, 2010. http://www.gotic.jp. Accessed April 4, 2016

19. Nakanishi Y, Kodama J, Tokumo K, Seki N, Miyagi Y, Yoshinouchi M, Okuda $\mathrm{H}$ and Kudo T: The expression of platelet-derived endothelial cell growth factor/thymidine phosphorylase associates with angiogenesis in epithelial ovarian cancer. Int J Clin Oncol 2: 19-23, 1997.

20. Suzuki M, Usui N, Furugen Y and Mitsuhasi N: Pyrimidine nucleoside phosphorylase activity in normal tissues of the uterus and ovary and in benign and malignant lesions of these organs. Int J Clin Oncol 6: 19-24, 2001.

21. Fujimoto J, Ichigo S, Sakaguchi H, Hirose R and Tamaya T: Expression of platelet-derived endothelial cell growth factor (PD-ECGF) and its mRNA in ovarian cancers. Cancer Lett 126: 83-88, 1998.

22. Seidman JD, Cho KR, Ronnett BM and Kurman RJ: Surface epithelial tumors of the ovary. In: Blaustein's Pathology of the Female Genital Tract. Kurman RJ, Ellenson LH and Ronnett BM (eds). 6th edition. Springer US, New York, NY, pp701-758, 2011.

23. Ogawa S, Kaku T, Kobayashi H, Hirakawa T, Ohishi Y, Kinukawa $\mathrm{N}$ and Nakano $\mathrm{H}$ : Prognostic significance of microvessel density, vascular cuffing and vascular endothelial growth factor expression in ovarian carcinoma: A special review for clear cell adenocarcinoma. Cancer Lett 176: 111-118, 2002.

24. Satou S, Nakajima A and Koyanagi Y: PhNPase-expressing macrophage and angiogenesis in colorectal cancer. Nippon Daicho Komonbyo Gakkai Zasshi 51: 267-275, 1998.

25. Konishi I: Angiogenic factors in gynecological cancer. Acta Obstet Gynaecol Jpn 52: 1222-1227, 2000.

26. Ogawa K, Katsube T, Konno S, Miura K, Wakasugi S, Watanabe T, Shimakawa $T$, Ishikawa $S$, Naritaka $Y$, Yagawa $\mathrm{H}$, et al: Influence of intratumor administration of OK-432 on the tumor selectivity of 5'-DFUR. Gan To Kagaku Ryoho 22: 2095-2100, 1995 (In Japanese).

27. Takao S, Akiyama SI, Nakajo A, Yoh H, Kitazono M, Natsugoe S, Miyadera K, Fukushima M, Yamada Y and Aikou T: Suppression of metastasis by thymidine phosphorylase inhibitor. Cancer Res 60: 5345-5348, 2000.

28. Mori S, Takao S, Ikeda R, Noma H, Mataki Y, Wang X, Akiyama $\mathrm{S}$ and Aikou T: Thymidine phosphorylase suppresses Fas-induced apoptotic signal transduction independent of its enzymatic activity. Biochem Biophys Res Commun 295: 300-305, 2002.

29. Overman MJ, Varadhachary G, Kopetz S, Thomas MB, Fukushima M, Kuwata K, Mita A, Wolff RA, Hoff PM, Xiong H and Abbruzzese JL: Phase 1 study of TAS-102 administered once daily on a 5-day-per-week schedule in patients with solid tumors. Invest New Drugs 26: 445-454, 2008. 